\title{
MRI-based Radiomics Model Can Improve the Predictive Performance of Postlaminar Optic Nerve Invasion in Retinoblastoma
}

\section{Zhenzhen Li}

Beijing Tongren Hospital

Jian Guo

Beijing Tongren Hospital

Xiaolin Xu

Beijing Tongren Hospital

Wenbin Wei

Beijing Tongren Hospital

Junfang Xian ( $\nabla$ cj.xianjunfang@vip.163.com )

Beijing Tongren Hospital https://orcid.org/0000-0003-2191-9393

\section{Research Article}

Keywords: Radiomics, magnetic resonance imaging (MRI), retinoblastoma, optic nerve

Posted Date: March 22nd, 2021

DOI: https://doi.org/10.21203/rs.3.rs-328789/v1

License: (1) This work is licensed under a Creative Commons Attribution 4.0 International License. Read Full License

Version of Record: A version of this preprint was published at The British Journal of Radiology on November 26th, 2021. See the published version at https://doi.org/10.1259/bjr.20211027. 


\section{Abstract}

Purpose: To develop an MRI-based radiomics model to predict postlaminar optic nerve invasion (PLONI) in retinoblastoma (RB) and to compare its predictive performance with that of subjective radiologists' assessment.

Methods: We retrospectively enrolled 124 patients with pathologically proven RB (90 in the training set and 34 in the validation set) who had MRI scans before surgery in this retrospective study. A radiomics model for predicting PLONI was developed by extracting 2058 quantitative imaging features from axial T2-weighted images and contrast-enhanced T1-weighted images in the training set. The Kruskal-Wallis test, least absolute shrinkage and selection operator regression, and recursive feature elimination were used for feature selection, whereupon a radiomics model was built with a logistic regression (LR) classifier. The area under the curve (AUC) of the receiver operating characteristic (ROC) curve and the accuracy were assessed to evaluate the predictive performance of PLONI in the training set and validation set. The performance of the radiomics model was compared to radiologists' assessment.

Results: The AUC of the radiomics model for the prediction of PLONI according to ROC analysis was 0.928 in the training set and 0.841 in the validation set. In all 124 patients, the AUC of the radiomics model was 0.897 , while that of radiologists' assessment was $0.674(p<0.001)$.

Conclusions: By incorporating MRI-based radiomics features, we constructed a radiomics model to predict PLONI in patients with RB, and it was shown to be superior to visual assessment and may serve as a potential tool to guide personalized treatment.

\section{Introduction}

Retinoblastoma (RB) is the most common primary intraocular malignancy in children, with an incidence of approximately 1 in 15,000 to 20,000 live births worldwide(1). Because of the dramatic evolution of RB management and treatment options, the cure rate exceeds $95 \%$ in developed countries, while it remains at $40 \%-80 \%$ in Asia and Africa(2-4). Distant metastasis is the principal cause of disease-related death among RB patients. The presence of postlaminar optic nerve invasion (PLONI) is a poor prognostic indicator and is associated with a higher likelihood of local recurrence or systemic metastasis(5-8). In recent years, substantial changes have taken place in terms of treatment regimens, with the increasing use of eye-sparing treatment strategies $(9,10)$. Although eye-salvage treatment has major advantages with regard to patient care, the lack of histopathologic examination for the detection of risk factors creates a challenge when making the decision whether to select enucleation or eye-sparing treatment. Therefore, accurate preoperative information about PLONI is crucial for identifying surgical candidates among those considered eye-sparing treatment strategies.

Magnetic resonance imaging (MRI) is an important technique to detect the presence of PLONI in patients who are considering eye-sparing treatment strategies(11-14). Currently, contrast-enhanced MRI is the imaging modality of choice for preoperative RB staging and a standard procedure in clinical practice(14). Many authors have reported the role of MRI in retinoblastoma and have shown an unsatisfactory efficacy at identifying PLONI, with a relatively limited accuracy of $52-79 \%$ and a relatively low sensitivity with a wide range 
$(37 \%-78 \%)(11-13,15-17)$. This low efficacy has led to a considerable proportion of patients being understated or overstated. Thus, the performance of noninvasive assessment by MRI has room for improvement. Radiomics uses the high-throughput extraction of advanced quantitative features to objectively and quantitatively describe tumor phenotypes, and it is gaining importance in cancer research $(18,19)$. These quantitative features, which may fail to be appreciated by the naked eye, can potentially provide valuable diagnostic, prognostic or predictive information in oncology(20). Recent studies(21-23) have shown that many radiomics features can significantly differentiate between early- and advanced-stage disease. To the best of our knowledge, there has been no MRI-based study on radiomics analysis for the preoperative prediction of PLONI in RB patients to date.

The purpose of the current study was to develop an MRI-based radiomics model with a logistic regression (LR) classifier to predict PLONI in RB patients and to compare its predictive performance with that of subjective radiologist assessment.

\section{Materials And Methods:}

\section{Patients}

This retrospective study adhered to the tenets of the Declaration of Helsinki and was approved by the local Ethics Committee of our hospital. The requirement for written informed consent was waived. Between January 2008 and January 2018, 124 consecutive patients with pathologically proven RB after enucleation were included in this study. The patient enrollment process for this study is shown in Fig. 1. The inclusion criteria were as follows: (a) all patients had an MRI scan of the orbit with pre- and postcontrast images, and (b) all MRI scans were performed within 4 months of enucleation. The exclusion criteria included (a) poor quality of MR images due to motion artifacts and (b) the RB patients who were negative in PLONI and had received shortterm treatment before enucleation. Among the 124 patients, nine patients had bilateral RB tumors, but only one tumor per patient was used in the data analysis (that with the shorter interval time between the MRI scan and enucleation). The pathological assessment of PLONI in the 124 RB patients showed that PLONI was confirmed in 54 globes and absent in 70 globes. Among the 54 RB patients with PLONI, 20 patients had received 1-3 cycles of CEV (carboplatin, etoposide, vindesine) intravenous chemotherapy (IVC). The median time between MR imaging and enucleation for the 20 patients was 54 days (range, 7-119 days). The median time between MR imaging and enucleation for the other 104 patients without any preoperative treatment was 16 days (range, $1-52$ days).

The consecutive study population was divided into two groups according to the time point. The training set (recruited from January 2008 to June 2015) consisted of 90 patients (37 with PLONI and 53 without PLONI). The validation set (recruited from July 2015 to January 2018) consisted of 34 consecutive patients (17 with PLONI and 17 without PLONI).

\section{MR image acquisition}

All MR images were obtained with a 1.5-Tesla (Signa Highspeed, GE Healthcare, Milwaukee, USA, $n=49$ ) or a 3Tesla (GE HDxt, GE Healthcare, Milwaukee, USA, n=46 or Discovery MR750; GE Healthcare, Milwaukee, WI, USA, 
$\mathrm{n}=29)$ scanner. Precontrast axial T1-weighted images (T1-WI), T2-weighted images (T2-WI) and postcontrast enhanced T1-WI (CET1-WI) in the axial and coronal planes were acquired for all 124 patients. The imaging parameters are shown in Table 1. CET1-WI was obtained after an intravenous bolus injection of $0.1 \mathrm{mmol} / \mathrm{kg}$ gadopentetate dimeglumine. Fat suppression (FS) was used in the axial CET1-WI.

Table 1

MR scanning parameters

\begin{tabular}{|c|c|c|c|c|c|c|c|c|}
\hline Sequence & $\begin{array}{l}\text { TR } \\
(\mathrm{ms})\end{array}$ & $\begin{array}{l}\text { TE } \\
(\mathrm{ms})\end{array}$ & $\begin{array}{l}\text { Field of } \\
\text { view }(\mathrm{mm})\end{array}$ & $\begin{array}{l}\text { Number } \\
\text { of slices }\end{array}$ & $\begin{array}{l}\text { Slice } \\
\text { thickness } \\
(\mathrm{mm})\end{array}$ & $\begin{array}{l}\text { Slice } \\
\text { gap } \\
(\mathrm{mm})\end{array}$ & NEX & Matrix \\
\hline \multirow[t]{2}{*}{ T1-WI } & \multirow{2}{*}{$\begin{array}{l}400- \\
500\end{array}$} & \multirow[t]{2}{*}{$8-11$} & \multirow[t]{2}{*}{$160 \times 160$} & \multirow[t]{2}{*}{16} & \multirow[t]{2}{*}{3.0} & \multirow[t]{2}{*}{0.3} & \multirow[t]{2}{*}{2} & $256 * 384(3.0 T)$ \\
\hline & & & & & & & & $244 * 288(1.5 T)$ \\
\hline \multirow[t]{2}{*}{$\mathrm{T} 2-\mathrm{WI}$} & \multirow{2}{*}{$\begin{array}{l}2860- \\
4440\end{array}$} & \multirow{2}{*}{$\begin{array}{l}115- \\
120\end{array}$} & \multirow[t]{2}{*}{$160 \times 160$} & \multirow[t]{2}{*}{16} & \multirow[t]{2}{*}{3.0} & \multirow[t]{2}{*}{0.3} & \multirow[t]{2}{*}{1} & $256 * 384(3.0 T)$ \\
\hline & & & & & & & & $244 * 288(1.5 T)$ \\
\hline \multirow[t]{2}{*}{ CET1-WI } & \multirow{2}{*}{$\begin{array}{l}400- \\
500\end{array}$} & \multirow[t]{2}{*}{ 8-11 } & \multirow[t]{2}{*}{$160 \times 160$} & \multirow[t]{2}{*}{16} & \multirow[t]{2}{*}{3.0} & \multirow[t]{2}{*}{0.3} & \multirow[t]{2}{*}{2} & $256 * 384(3.0 T)$ \\
\hline & & & & & & & & $244 * 288(1.5 T)$ \\
\hline
\end{tabular}

\section{Region-of-interest segmentation and radiomics feature extraction}

Manual segmentation for each of the 124 RB tumors was performed by two radiologists (Radiologist 1 and Radiologist 2, with 3 years and 15 years of experience, respectively, in reading head and neck images). The segmented region of interest (ROI) covered the whole tumor and was delineated by the radiologists on both the axial T2-WI and CET1-WI on each slice. For each MR sequence, 1029 radiomics features were extracted on the Radcloud platform, which is a useful tool for extracting radiomics features with a large panel of engineered hard-coded feature algorithms (Huiying Medical Technology Co., Ltd. http://mics.radcloud.cn/)(24, 25). The 1029 obtained features can be divided into four main categories: first order, shape feature, texture feature, and higher-order statistical features. First-order statistics such as the mean, standard deviation, variance, maximum, median, and range describe the intensity information in the MRI region of interest. Shape features such as volume, surface area, compactness, and maximum diameter reflect the shape and size of the region. Texture features can quantify the regional differences in heterogeneity. The following higher-order statistical features included the first-order statistics and texture features derived from wavelet transformation of the original images: exponential, square, square root, logarithm and wavelet (wavelet-LHL, wavelet-LHH, wavelet$H L L$, wavelet-LLH, wavelet-HLH, wavelet-HHH, wavelet-HHL, and wavelet-LLL). These features complied with 
the definitions produced by the Imaging Biomarker Standardization Initiative. We used the Radcloud platform to manage the imaging data and perform subsequent radiomics statistical analyses.

Different medical imaging factors cause inconsistencies in the image intensity information of tissues of the same nature. We used the following formula for intensity normalization (where $\mathrm{x}$ indicates the original intensity; $f(x)$ indicates the normalized intensity; $\mu$ refers to the mean value; $\sigma$ indicates the variance; $s$ is an optional scaling, and by default, it is set to 1 ).

$$
f(x)=\frac{s\left(x-\mu_{x}\right)}{\sigma_{x}}
$$

\section{Inter- and intraobserver reproducibility evaluation}

Interobserver and intraobserver reproducibility of ROI detection and radiomics feature extraction was initially determined by two radiologists using the T2-WI and CET1-WI data of 20 patients chosen by computergenerated random numbers. To assess intraobserver reproducibility, Radiologist 1 repeated the generation of radiomics features twice within a 1-month period following the same procedure.

\section{Feature selection and model construction}

Feature selection and model construction were only performed on the training set, and the validation set was only used to evaluate the model performance. We applied the Kruskal-Wallis (K-W) test to compare the distributions of feature values across three different MR scanners, and features with $\mathrm{P}<0.05$ were removed to reduce redundant features and avoid collinearity and overfitting. Then, the least absolute shrinkage and selection operator (LASSO) logistic regression algorithm was used to reduce the dimensionality of extracted features on the training set. The initial alpha was set to 0.1 , and the tuning parameter $\lambda$ was set to zero as the default. Finally, the recursive feature elimination (RFE) with the SVM (kernel: linear) estimator was performed as a multivariate analysis method to select the PLONI-related features. RFE constantly eliminates unimportant features to obtain the optimal feature set by calculating the importance of features. Logistic regression (LR) classifier analysis was conducted to develop a model for the prediction of PLONI in the training set. The performance of the radiomics model was then internally tested in an independent validation set with the formula derived from the training set. The radiomics workflow is shown in Fig. 2.

\section{Radiologist assessment}

In this study, abnormal enhancement of the optic nerve in continuity with the tumor on postcontrast MR images regardless of the asymmetrical thickening of the nerve was defined as MRI-based PLONI in RB patients. The same two radiologists who were blinded to the pathologic findings classified the 124 patients into PLONI-positive or PLONI-negative groups based on their imaging interpretations. The performance of 
radiologist assessment was compared with that of the radiomics model with regard to the prediction of PLONI in RB patients.

\section{Statistical analysis}

Univariate and bivariate analyses were performed with SPSS version 20 (SPSS Inc.). Statistical analyses were performed for both the training and validation sets. Student's t-test and the chi-square test were used to assess differences in age and sex distributions between the training and validation sets. Dice's coefficients were used to evaluate the intra- and interobserver consistency for the ROI segmentation and radiomics feature extraction with 20 randomly selected samples. We interpreted a coefficient of 0.81 to 1.00 as almost perfect agreement, 0.61 to 0.80 as substantial agreement, 0.41 to 0.60 as moderate agreement, 0.21 to 0.40 as fair agreement, and 0 to 0.20 as poor or no agreement. Receiver operating characteristic (ROC) analysis and the area under the curve (AUC) were used to assess the predictive performance of the radiomics model and visual assessment for PLONI. The cutoff values of the ROC curves were determined by the principle of maximizing the Youden index. The AUC of the radiomics model was compared with that of visual assessment by the DeLong test. $P<0.05$ was considered to indicate a statistically significant difference.

\section{Results}

\section{Clinical information in data analysis}

The training set included 90 patients who were recruited between January 2008 and June 2015, and the validation set included 34 patients who were recruited between July 2015 and January 2018. Only patient sex and age were used in the data analysis (Table 2). There were no significant differences between the two groups in gender and age in either the training set or validation set $(p>0.05)$.

Table 2

Gender and age distribution of training and validation set

\begin{tabular}{|c|c|c|c|c|c|c|}
\hline & \multicolumn{3}{|l|}{ Training set } & \multicolumn{3}{|c|}{ Validation set } \\
\hline & $\begin{array}{l}\text { With } \\
\text { PLONI }(n= \\
37)\end{array}$ & $\begin{array}{l}\text { Without } \\
\text { PLONI }(n=53)\end{array}$ & $\begin{array}{l}P \\
\text { value }\end{array}$ & $\begin{array}{l}\text { With } \\
\text { PLONI(n = } \\
\text { 17) }\end{array}$ & $\begin{array}{l}\text { Without } \\
\text { PLONI }(n=17)\end{array}$ & $\begin{array}{l}P \\
\text { value }\end{array}$ \\
\hline \multicolumn{7}{|l|}{ Gender } \\
\hline Male & 21 & 36 & 0.279 & 11 & 10 & 0.724 \\
\hline Female & 16 & 17 & & 6 & 7 & \\
\hline $\begin{array}{l}\text { Age (mean } \pm S D \text {, } \\
\text { months) }\end{array}$ & $30.56 \pm 8.07$ & $37.38 \pm 22.69$ & 0.376 & $\begin{array}{l}32.81 \pm \\
16.89\end{array}$ & $34.75 \pm 19.10$ & 0.620 \\
\hline
\end{tabular}

\section{Imaging feature selection and radiomics model development}

Satisfactory interobserver and intraobserver reproducibility was achieved for radiomics feature extraction. The mean Dice's coefficients were greater than 0.81 . 
A total of 2058 features were extracted from MR images (1029 features from T2-WI and 1029 features from CET1-WI). First, the $\mathrm{K}-\mathrm{W}$ test was performed to separate the relevant and redundant features, and 54 features from T2-WI and 136 features from CET1-WI remained. After applying the LASSO and RFE methods, we selected the three features from T2-WI and the six features from CET1-WI that were most strongly associated with PLONI in the training set (Table 3). To build the radiomics signature, the above nine features were selected for inclusion in the Rad-score prognostic model. Rad-scores for each patient in the training and validation sets are shown in Fig. 3.

Table 3

Nine selected MRI features for inclusion in the Rad-score prognostic model

\begin{tabular}{|c|c|c|c|}
\hline $\begin{array}{l}\text { Imaging } \\
\text { technique }\end{array}$ & T2-w & CET1-w & T2-w + CET1-w \\
\hline \multirow[t]{9}{*}{ Imaging feature } & & wavelet-HLH_glrlm_GLNU & CE_wavelet-HLH_glrlm_GLNU \\
\hline & & original_shape_Max3D & CE_original_shape_Max3D \\
\hline & & exponential_glszm_SAE & CE_exponential_glszm_SAE \\
\hline & & $\begin{array}{l}\text { wavelet-HHH_first order } \\
\text { _Skewness }\end{array}$ & $\begin{array}{l}\text { CE_wavelet-HHH_first order } \\
\text { _Skewness }\end{array}$ \\
\hline & & $\begin{array}{l}\text { wavelet-LLH_first order } \\
\text { _Skewness }\end{array}$ & $\begin{array}{l}\text { CE_wavelet-LLH_first order } \\
\text { _Skewness }\end{array}$ \\
\hline & & wavelet-LHL_glcm_IV & CE_wavelet-LHL_glcm_IV \\
\hline & $\begin{array}{l}\text { wavelet-HHL_ first order } \\
\text { _Median }\end{array}$ & & $\begin{array}{l}\text { T2-w_wavelet-HHL_ first order } \\
\text { _Median }\end{array}$ \\
\hline & original_shape_MinAxis & & T2-w_original_shape_MinAxis \\
\hline & original_shape_SA & & T2-w_original_shape_SA \\
\hline $\begin{array}{l}\text { intercept (non- } \\
\text { feature) }\end{array}$ & -0.534 & -0.498 & -0.621 \\
\hline \multicolumn{4}{|c|}{$\begin{array}{l}\text { CET1-w = contrast-enhanced T1 weighted; } \text { T2-w = T2-weighted; } \text { coef }=\text { coefficients; } \text { MinAxis = Minor Axis; SA } \\
\text { = Surface Area; GLNU = Gray Level Non Uniformity; Max3D = Maximum3DDiameter; SAE = Small Area } \\
\text { Emphasis; IV = Inverse Variance }\end{array}$} \\
\hline
\end{tabular}

In the training set, the radiomics signature derived from CET1-WI yielded an AUC of 0.844 [95\% confidence interval (CI): 0.611-0.926]. The radiomics signature from T2-WI yielded an AUC of 0.860 ( $95 \% \mathrm{Cl}$ : $0.781-$ 0.940). In the validation set, the radiomics signature from CET1-WI yielded an AUC of 0.768 ( $95 \%$ Cl: $0.544-$ 0.904). The radiomics signature from T2-WI yielded an AUC of 0.830 (95\% Cl: $0.687-0.974)$. The radiomics signature derived from both CET1-WI and T2-WI yielded the highest AUC both in the training set and validation set: 0.928 (95\% Cl: 0.873-0.982) and 0.841 (95\% Cl: 0.707-0.974), respectively. A final radiomics model was developed based on the combination of CET1-WI and T2-WI features.

\section{Performance comparison between the radiomics model and radiologist assessment}


The overall performance of the radiomics model compared with that of the radiologist assessment is shown in Table 4. In all patients, the radiomics signature from the combined CET1-WI and T2-WI features yielded an AUC of 0.897 (95\% Cl: $0.839-0.955)$. The AUC value for the radiologist assessment was 0.674 ( $95 \% \mathrm{Cl}: 0.576-$ 0.773). In the ROC analysis, the AUC values of the radiomics model were significantly higher than those of the radiologist assessment in the training set and in all 124 patients (both $p<0.001$ ) (Fig. 4).

Table 4

Overall performance of radiomics model compared with radiologist assessment in predicting PLONI

\begin{tabular}{|c|c|c|c|c|c|c|}
\hline & \multicolumn{3}{|c|}{ Radiomics model } & \multicolumn{3}{|c|}{ Radiologist assessment } \\
\hline & Primary set & $\begin{array}{l}\text { Validation } \\
\text { set }\end{array}$ & All cases & Primary set & $\begin{array}{l}\text { Validation } \\
\text { set }\end{array}$ & All cases \\
\hline $\begin{array}{l}\text { Sensitivity } \\
(\%)(n)\end{array}$ & $81.1(30 / 37)$ & $82.4(14 / 17)$ & $81.5(44 / 54)$ & $43.2(16 / 37)$ & $52.9(9 / 17)$ & $46.3(25 / 54)$ \\
\hline $\begin{array}{l}\text { Specificity } \\
(\%)(n)\end{array}$ & $94.3(50 / 53)$ & $76.5(13 / 17)$ & $90.0(63 / 70)$ & $88.7(47 / 53)$ & $88.2(15 / 17)$ & $88.6(62 / 70)$ \\
\hline $\begin{array}{l}\text { Accuracy } \\
(\%)(n)\end{array}$ & $88.9(80 / 90)$ & $79.4(27 / 34)$ & $86.3(107 / 124)$ & $70.0(63 / 90)$ & $70.6(24 / 34)$ & $70.2(87 / 124)$ \\
\hline $\begin{array}{l}\mathrm{PPV}(\%) \\
(\mathrm{n})\end{array}$ & $90.9(30 / 33)$ & $77.8(14 / 18)$ & $86.3(44 / 51)$ & $72.7(16 / 22)$ & $82.8(9 / 11)$ & $75.8(25 / 33)$ \\
\hline $\begin{array}{l}\mathrm{NPV}(\%) \\
(\mathrm{n})\end{array}$ & $87.7(50 / 57)$ & $81.3(13 / 16)$ & $86.3(63 / 73)$ & $69.1(47 / 68)$ & $65.2(15 / 23)$ & $68.1(62 / 91)$ \\
\hline AUC & 0.928 & 0.841 & 0.897 & 0.660 & 0.706 & 0.675 \\
\hline
\end{tabular}

Moreover, all eight cases wrongly classified as PLONI by radiologists were correctly predicted by the radiomics model. Among 28 false negative cases classified by radiologists, 20 cases were correctly classified by the radiomics model. Two image examples are shown in Fig. 5 and Fig. 6.

\section{Discussion}

PLONI is considered to be one of the most important risk factors for metastasis and recurrence, which may alter treatment plans. Although histopathologic examination is the gold standard for identifying PLONI, MRI is the only method of detecting the presence of PLONI in patients undergoing eye-saving treatments(26). Previous studies also investigated that some MRI features, such as the presence of optic nerve enhancement and tumor size, could be used to predict PLONI $(12,13)$. Abnormal optic nerve enhancement on CET1-WI is widely regarded as a useful imaging parameter for detecting PLONI in clinical practice. MRI still has some limitations with regard to reliably predicting PLONI, and the diagnostic efficiency, especially the sensitivity, is inadequate $(15,17)$. A recent meta-analysis by De Jong et al. (13)reported the relatively low sensitivity $(59 \%)$ of the detection of PLONI by MRI. In our study, the predictive performance of the radiologist assessment, which was based on the nerve enhancement parameter on postcontrast MRI, showed a similar low sensitivity of $46 \%$. Therefore, the usefulness of MRI for the identification of PLONI had some defects in clinical use. In this study, we developed an MRI-based radiomics model for the prediction of PLONI, which was proven to have much 
better predictive performance than the radiologist assessment (AUC values of 0.90 versus 0.68 , sensitivity of 81.5 versus 46.3 , respectively, for all 124 patients).

In this study, we found that all eight false positive cases classified by radiologists were correctly classified by the radiomics model, and 20 (71\%) of the 28 false negatives classified by radiologists were correctly classified by the radiomics model. The sensitivity and negative predictive value (NPV) of the radiomics model were much better than those of the radiologist assessment for the prediction of PLONI. The radiomics model accurately excludes PLONI, as reflected in the high NPV. In the current era of increasingly conservative treatment strategies for diseases, including advanced-stage retinoblastoma, ophthalmologists' confidence in the use of conservative treatment strategies would be improved by this higher NPV. The higher sensitivity can help inform the choices made by patients' families, and the option of primary enucleation can be offered to avoid delaying cancer treatment(9). A choice of many treatments is available to ophthalmologists, and several factors play a role in determining the initial treatment strategy for this cancer. We believe that this MRI-based radiomics model has the potential to be used by radiologists to improve their diagnostic performance with regard to predicting PLONI and patient management.

The radiomics signature derived from CET1-WI and T2-WI showed promising predictive performance for the detection of PLONI in both the training set (AUC of 0.93) and the validation set (AUC of 0.84). Nine descriptive radiomics features were enrolled in the model, some of which were also found by other authors to be useful in the evaluation of head and neck lesions, such as skewness and Max3D(27-29). Most features selected for inclusion in the radiomics model were higher-order statistics features and filtered wavelet features.

Morphological and obvious textural features of tumors can be easily identified by radiologists, while highdimensional radiomics features are almost impossible to decipher with the naked eye, which may explain why the radiomics model performed better than did visual assessment. One previous radiomics study ${ }^{30}$ on ovarian cancer found that the skewness was significantly greater in the malignant group, signifying that more heterogeneity and clustering were associated with the development of cancer. Positive skewness was also reported to be significantly associated with more distant metastases in lung cancer(31-33). This is consistent with the findings of other investigators. Our results also indicated that positive skewness was associated with PLONI.

Our study had several limitations. First, the number of patients was small relative to the high dimensionality of the radiomics features. This is because in the current era of the increasing use of conservative eye-sparing treatment strategies for RB, increasingly fewer primary enucleations of the affected eye are being performed in RB patients. Second, high-resolution MR imaging was not used due to the retrospective nature of the study. However, the sensitivity, specificity, and accuracy of our study involving visual assessment were similar to those of a study using high-resolution MRI(12) (sensitivity, specificity, accuracy: $0.46,0.89,0.70$ vs $0.42,0.85$, 0.60). Third, an external validation of the model should be applied in the further study to improve the reliability of result, due to all the patients from a single center in this study. More clinical information, such as ICRB and a prolonged duration of symptoms, was not used in the data analysis. Finally, this was a retrospective study, and the utility of the model needs to be investigated in a prospective study.

In conclusion, this MRI-based radiomics model is superior to radiologist assessment for the prediction of PLONI in RB patients and can potentially help oncologists improve patient management. 


\section{Declarations \\ Funding】}

This study was supported by Beijing Municipal Administration of Hospital Clinical Medicine Development of Special Funding Support (ZYLX201704) and Beijing Municipal Administration of Hospitals'Ascent Plan (DFL20190203).

\section{Conflict of interest:}

No conflicting relationship exists for any author.

\section{Availability of data and material:}

The datasets analyzed during the current study are available from the corresponding author on reasonable request.

\section{Code availability:}

Huiying Medical Technology Co., Ltd. http://mics.radcloud.cn/.

\section{Authors' contributions:}

Conceptualization: Wenbin Wei.

Data curation: Zhenzhen Li.

Formal analysis: Zhenzhen Li, Jian Guo.

Investigation: Zhenzhen Li, Jian Guo.

Methodology: Jian Guo, Junfang Xian.

Project administration: Junfang Xian.

Software: Zhenzhen Li.

Resources: Xiaolin Xu.

Supervision: Wenbin Wei.

Writing - original draft: Zhenzhen Li.

Writing - review \& editing: Jian Guo. 


\section{Ethics approval:}

This study was approved by the institutional ethics review board.

\section{Consent to participate:}

No informed consent was required as per retrospective and anonymized nature of the image datasets.

\section{Consent for publication:}

The authors agree to publication in the Journal.

\section{References}

1. Kivela $T$ (2009) The epidemiological challenge of the most frequent eye cancer: retinoblastoma, an issue of birth and death. Brit J Ophthalmol 93(9):1129-31. https://doi.org/10.1136/bjo.2008.150292

2. Broaddus E, Topham A, Singh AD (2009) Incidence of retinoblastoma in the USA: 1975-2004. Brit J Ophthalmol 93(1): 21-4. https://doi.org/10.1136/bjo.2008.138750

3. Bowman RJ, Mafwiri M, Luthert P, Luande J, Wood M (2010) Outcome of retinoblastoma in east Africa. Pediatr Blood Cancer. 2010;50(1):160-2. https://doi.org/10.1002/pbc.21080

4. Helen D, Kahaki K, Dimba EAO et al (2012) Retinoblastoma. Lancet 379(9824):1436-46. https://doi.org/10.1016/S0140-6736(11)61137-9

5. Kaliki S, Shields C L, Rojanaporn D et al (2013) High-Risk Retinoblastoma Based on International Classification of Retinoblastoma: Analysis of 519 Enucleated Eyes. Ophthalmology 2013;120(5):9971003. https://doi.org/10.1016/j.ophtha.2012.10.044

6. Khelfaoui F, Validire P, Auperin A et al (1996) Histopathologic risk factors in retinoblastoma: a retrospective study of 172 patients treated in a single institution. Cancer-Am Cancer Soc 77(6):1206-13. https://doi.org/10.1002/(SICI)1097-0142(19960315)77:6<1206::AID-CNCR30>3.0

7. Messmer EP, Heinrich T, Höpping W et al (1991) Risk factors for metastases in patients with retinoblastoma. Ophthalmology 98(2):136-41. https://doi.org/10.1016/S0161-6420(91)32325-X

8. Shields CL, Shields JA, Baez K, Cater JR, De Potter P. Optic nerve invasion of retinoblastoma. Metastatic potential and clinical risk factors. Cancer-Am Cancer Soc 1994;73(3):692-8. https://doi.org/10.1002/10970142(19940201)73:3<692::AID-CNCR2820730331>3

9. Fabian I D, Onadim Z, Karaa E et al (2018) The management of retinoblastoma. Oncogene 37(12):155160. https://doi.org/10.1038/s41388-017-0050-x

10. Radhakrishnan V, Kashyap S, Pushker N et al (2012) Outcome, Pathologic Findings, and Compliance in Orbital Retinoblastoma (International Retinoblastoma Staging System Stage III) Treated with Neoadjuvant Chemotherapy. Ophthalmology 119(7):1470-7. https://doi.org/10.1016/j.ophtha.2012.01.029

11. De Jong M C, de Graaf P, Noij D P et al (2014) Diagnostic Performance of Magnetic Resonance Imaging and Computed Tomography for Advanced Retinoblastoma. Ophthalmology 121(5):1109-18. 
https://doi.org/10.1016/j.ophtha.2013.11.021

12. Brisse H J, de Graaf $P$, Galluzzi $P$ et al (2015) Assessment of early-stage optic nerve invasion in retinoblastoma using high-resolution 1.5 Tesla MRI with surface coils: a multicentre, prospective accuracy study with histopathological correlation. Eur Radiol 25(5):1443-52. https://doi.org/10.1007/s00330-0143514-1

13. De Jong M C, van der Meer F J, Goricke S L et al (2016) Diagnostic Accuracy of Intraocular Tumor Size Measured with MR Imaging in the Prediction of Postlaminar Optic Nerve Invasion and Massive Choroidal Invasion of Retinoblastoma. Radiology 279(3):817-26. https://doi.org/10.1148/radiol.2015151213

14. De Graaf P, Göricke S, Rodjan F et al (2012) Guidelines for imaging retinoblastoma: imaging principles and MRI standardization. Pediatr Radiol 42(1):2-14. https:// doi.org/10.1007/s00247-011-2201-5

15. Sirin S, Schlamann M, Metz K A et al (2013) Diagnostic image quality of gadolinium-enhanced T1weighted MRI with and without fat saturation in children with retinoblastoma. Pediatr Radiol 43(6):716-24. https://doi.org/10.1007/s00247-012-2576-y

16. Sirin S, Schlamann M, Metz K A et al (2015) High-resolution MRI using orbit surface coils for the evaluation of metastatic risk factors in 143 children with retinoblastoma. Neuroradiology 57(8):815-24. https://doi.org/10.1007/s00234-015-1544-2

17. Song KD, Eo H, Kim JH, Yoo S, Jeon TY (2012) Can preoperative MR imaging predict optic nerve invasion of retinoblastoma? Eur J Radio/ 81(12):4041-5. https://doi.org/10.1016/j.ejrad.2012.03.034

18. Lambin P, Leijenaar R T H, Deist T M et al (2017) Radiomics: the bridge between medical imaging and personalized medicine. Nat Rev Clin Onco/14(12):749-62. https://doi.org/10.1038/nrclinonc.2017.141

19. Gillies RJ, Kinahan PE, Hricak H (2016) Radiomics: Images Are More than Pictures, They Are Data. Radiology 278(2):563-77. https://doi.org/10.1148/radiol.2015151169

20. Yip SS, Aerts HJ (2016) Applications and limitations of radiomics. Phys Med Biol 61(13):R150-66. https://doi.org/10.1088/0031-9155/61/13/R150

21. Mu W, Chen Z, Liang Y et al (2015). Staging of cervical cancer based on tumor heterogeneity characterized by texture features on F-18-FDG PET images. Phys Med. Biol. 60(13):5123-39. https://doi.org/10.1088/0031-9155/60/13/5123

22. Dong X, Xing L, Wu P et al (2013) Three-dimensional positron emission tomography image texture analysis of esophageal squamous cell carcinoma: relationship between tumor 18F-fluorodeoxyglucose uptake heterogeneity, maximum standardized uptake value, and tumor stage. Nucl Med Commun 34(1):40-6. https://doi.org/10.1097/MNM.0b013e32835ae50c

23. Huang Y Q, Liang C H, He L et al (2016) Development and Validation of a Radiomics Nomogram for Preoperative Prediction of Lymph Node Metastasis in Colorectal Cancer. J Clin Oncol34(18):2157-64. https://doi.org/10.1200/JC0.2015.65.9128

24. Bektas C T, Kocak B, Yardimci A H et al (2017) Clear Cell Renal Cell Carcinoma: Machine Learning-Based Quantitative Computed Tomography Texture Analysis for Prediction of Fuhrman Nuclear Grade. Eur Radiol 29, 1153-1163. https://doi.org/10.1007/s00330-018-5698-2

25. Liang M, Cai Z, Zhang H, Huang C, Zhao X (2019) Machine Learning-based Analysis of Rectal Cancer MRI Radiomics for Prediction of Metachronous Liver Metastasis. Acad Radiol 26(11):1495-1504 https://doi.org/10.1016/j.acra.2018.12.019. 
26. Kaliki S, Srinivasan V, Gupta A, Mishra DK, Naik MN (2015) Clinical features predictive of high-risk retinoblastoma in 403 Asian Indian patients: a case-control study. Ophthalmology 122(6):1165-72. https://doi.org/10.1016/j.ophtha.2015.01.018

27. Zhang B, Tian J, Dong D et al (2017) Radiomics Features of Multiparametric MRI as Novel Prognostic Factors in Advanced Nasopharyngeal Carcinoma. Clin Cancer Res 23(15):4259-69. https://doi.org/10.1158/1078-0432.CCR-16-2910

28. Guo J, Liu Z, Shen C et al (2018) MR-based radiomics signature in differentiating ocular adnexal lymphoma from idiopathic orbital inflammation. Eur Radiol 28(9):3872-81. https://doi.org/10.1007/s00330-018-5381-7

29. Peng H, Dong D, Fang M J et al (2019) Prognostic Value of Deep Learning PET/CT-Based Radiomics: Potential Role for Future Individual Induction Chemotherapy in Advanced Nasopharyngeal Carcinoma. Clin Cancer Res 25(14):4271-9. https://doi.org/10.1158/1078-0432.CCR-18-3065

30. Nandy S, Sanders M, Zhu Q (2016) Classification and analysis of human ovarian tissue using full field optical coherence tomography. Biomed Opt Express 7(12):5182-5187. https://doi.org/10.1364/BOE.7.005182

31. Weiss G J, Ganeshan B, Miles K A et al (2014) Noninvasive Image Texture Analysis Differentiates K-ras Mutation from Pan-Wildtype NSCLC and Is Prognostic. Plos One. 9(7): e100244 Available online: https://doi.org/ 10.1371/journal.pone.010024.

32. Oikonomou A, Khalvati F, Tyrrell P N et al (2018) Radiomics analysis at PET/CT contributes to prognosis of recurrence and survival in lung cancer treated with stereotactic body radiotherapy. Sci Rep 8(1):400314. https://doi.org/10.1038/s41598-018-22357-y

33. Li M, Li X, Guo Y et al (2020) Development and assessment of an individualized nomogram to predict colorectal cancer liver metastases. Quant Imaging Med Surg. 10(2):397-414. https://doi.org/10.21037/qims.2019.12.16

\section{Figures}




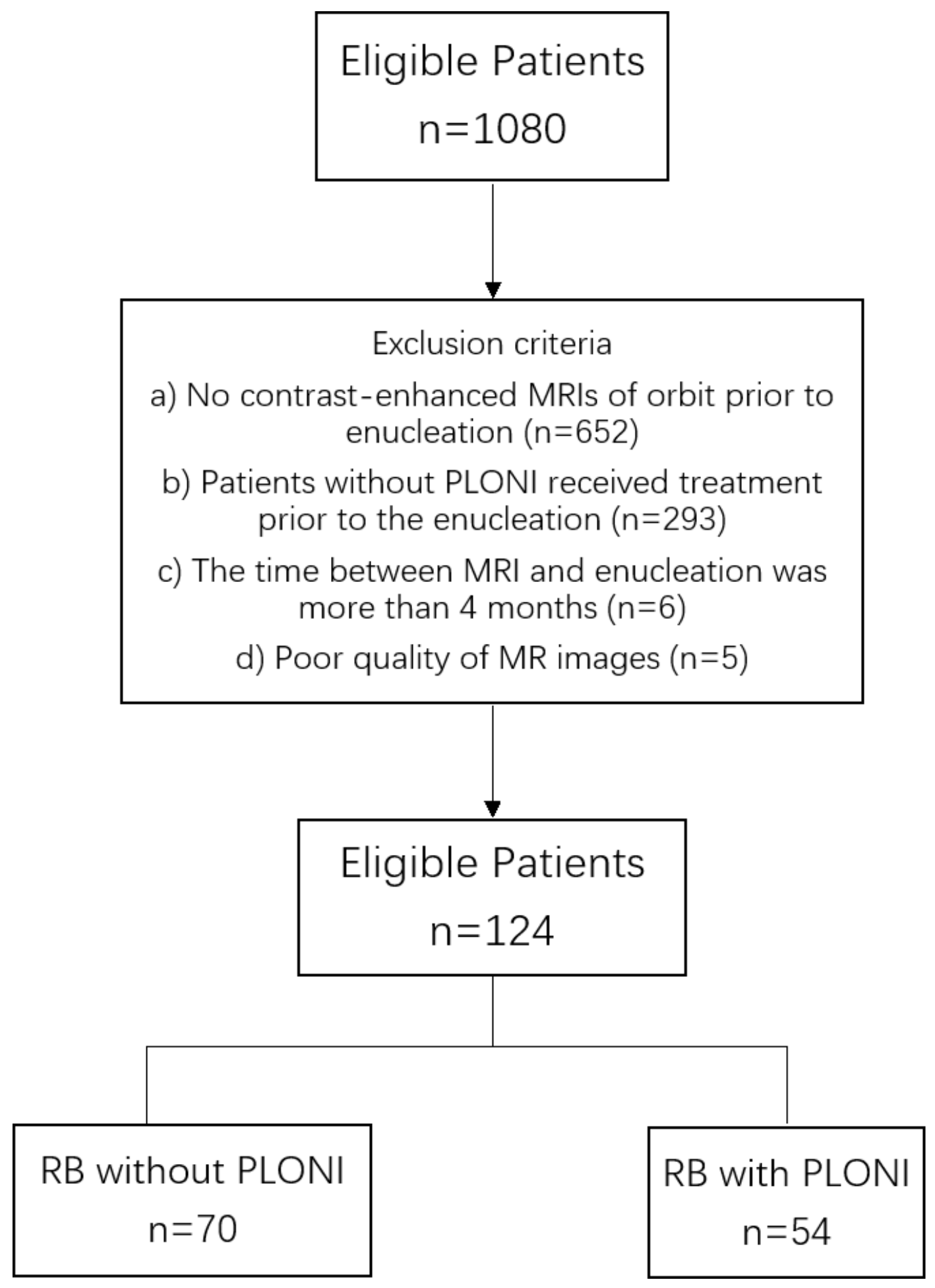

Figure 1

Flowchart of the patient enrolment process. PLONI postlaminar optic nerve invasion. RB: retinoblastoma 


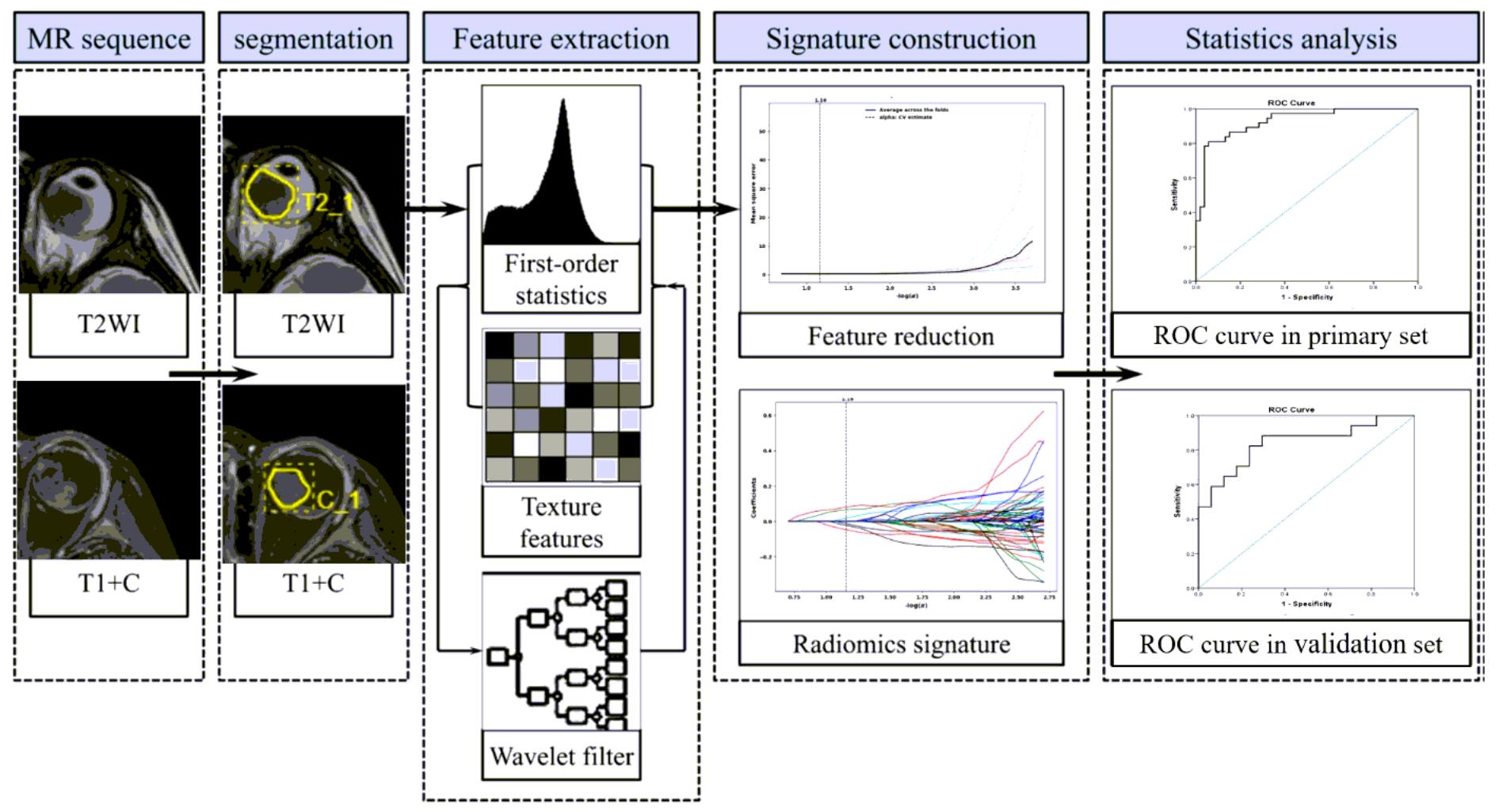

Figure 2

The radiomics workflow.
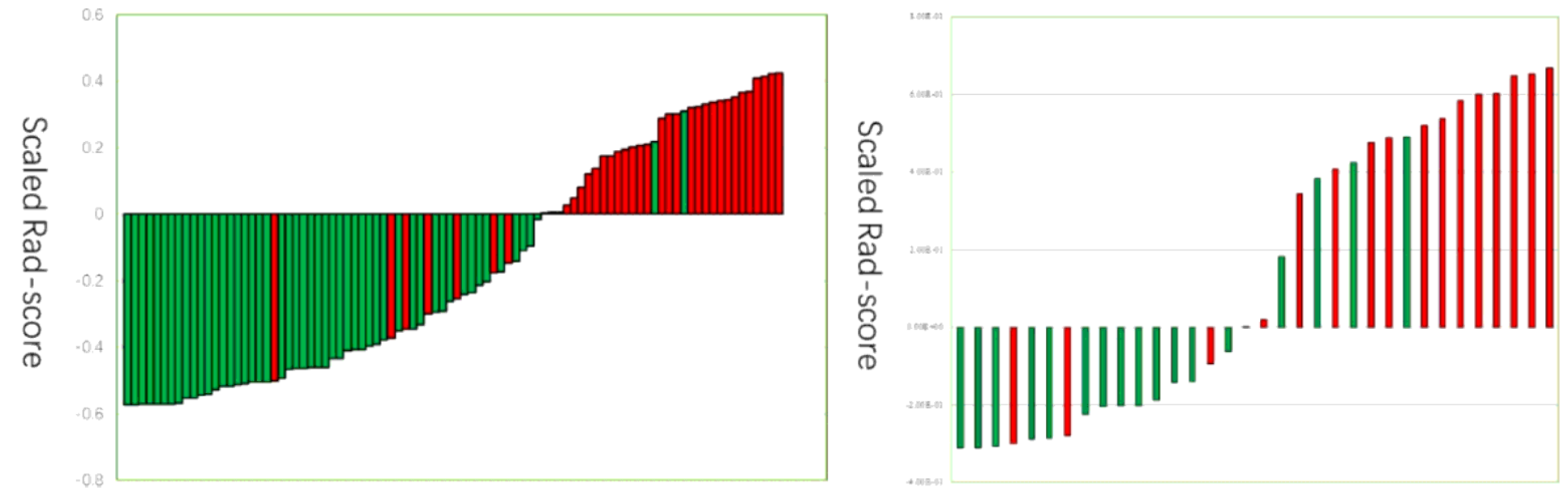

Figure 3

Rad-score for each patient in the training (a) and validation cohort (b). The red bar with a Rad-score $>0$ indicates that the signature successfully classifies the RB patients with PLONl; the red bar with a Rad-score $<0$ indicates that the signature fails to the RB patients with PLONI. For the green bar, the contrary applies. 
a

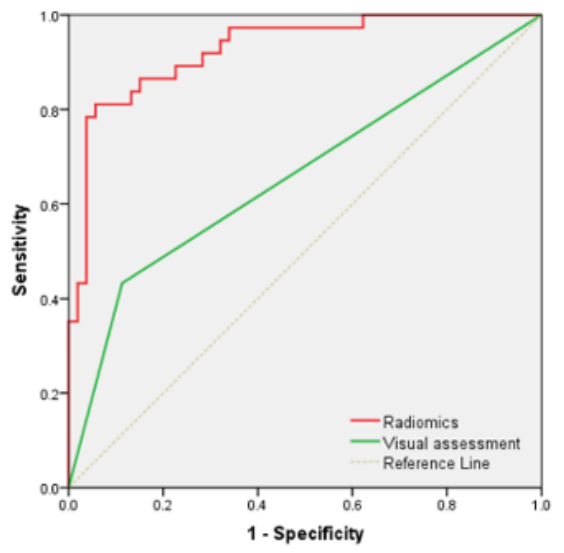

b

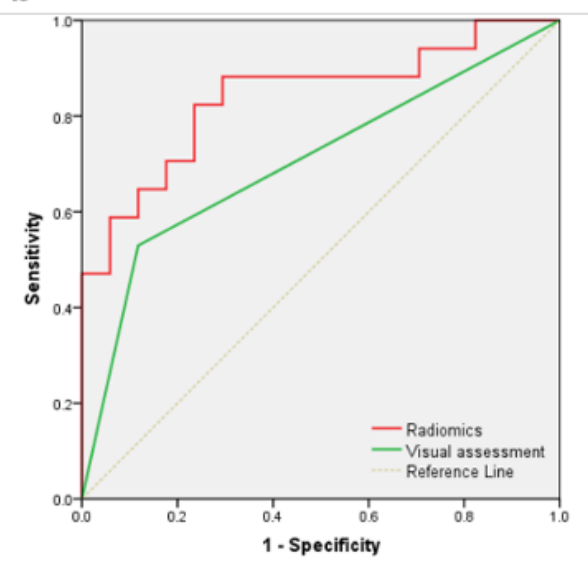

C

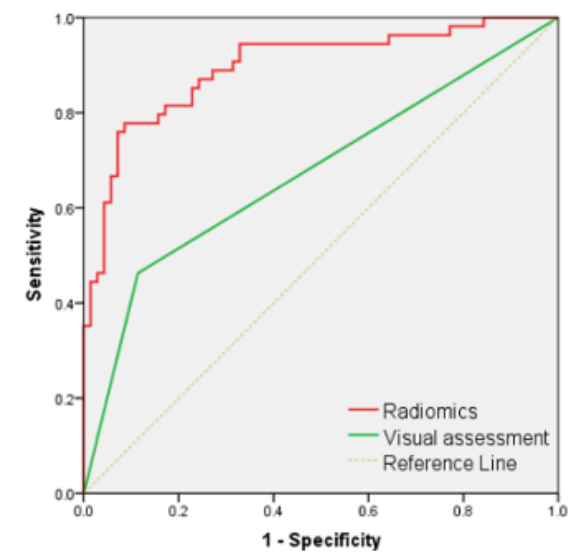

\section{Figure 4}

Receiver operating characteristic (ROC) curves for the prediction of PLONI in RB by the radiomics model and radiologist assessment in the training set (a), validation set (b), and all patients (c). The areas under the ROC curve (AUCs) for the radiomics model were 0.928 (a), 0.841 (b) and 0.897 (c), respectively. The AUCs for radiologist assessment were 0.660 (a), 0.706 (b), and 0.675 (c), respectively. The AUCs for the radiomics model were significantly higher than those for radiologist assessment in the training set and in all 124 patients $(p<0.001)$. 


\section{Figure 5}

Retinoblastoma in a 36-month-old male child without histopathological PLONI. CET1-WI with fat suppression shows an irregular tumor in the left globe with focal enhancement at the nerve-globe junction (arrow). It was misclassified as a positive case by radiologist assessment and was correctly classified by the radiomics model.
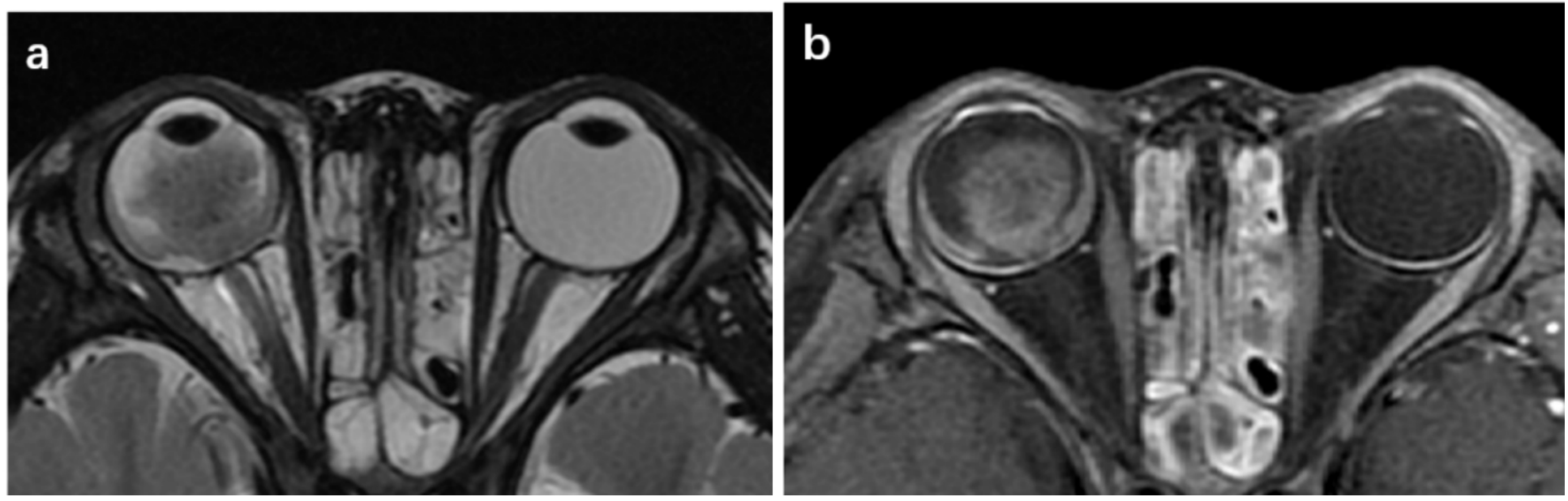

Figure 6

Retinoblastoma in a 45-month-old male child with pathologically confirmed PLONI. Axial T2-WI (a) and axial CET1-WI with fat suppression (b) shows a large irregular tumor in the right globe. The lesion showed slight heterogeneous enhancement without obvious enhancement in the postlaminar optic nerve on CET1-WI. It was misclassified as a negative case by radiologist assessment and was correctly classified by the radiomics model. 